\title{
Is Facebook Easier to Use than WeChat?
}

\author{
A Critical Comparative Analysis of Interface Features of WeChat and Facebook
}

\author{
Shyam B. Pandey \\ English Department, Purdue University, West Lafayette, \\ Indiana, USA \\ pandey24@purdue.edu
}

\begin{abstract}
Given the dominant role that Facebook and WeChat play in their respective contexts, researchers have explored users' behavior and uses of these two globally leading social networking sites (SNSs). However, there is scant research comparing the interface features of these two SNSs and their contextual interactions within and across cultures. Utilizing Sun's "culturally localized user experience" (CLUE) framework [27, 28], this study employs critical data analysis to investigate the defining interface features of Facebook and WeChat in four sections of moments/status, official accounts, digital currency, and group chat. Our study expands the understanding of cultural considerations in interface design and encourages factoring contextualized use of technology and the sociological approach to interface design. The findings reveal cultural and local differences are the driving force for the unique interface features of the two SNSs and highlight "glocalization" (mixing forces into local elements) in SNS design [27].
\end{abstract}

\section{CCS CONCEPTS}

- Human-centered computing; • Interaction design; • Interaction design process and methods; • User interface design;

\section{KEYWORDS}

Interface design, Facebook, WeChat, critical comparative analysis, culturally localized user experience

\section{ACM Reference Format:}

Shyam B. Pandey and Jianfen Chen. 2021. Is Facebook Easier to Use than WeChat?: A Critical Comparative Analysis of Interface Features of WeChat and Facebook. In The 39th ACM International Conference on Design of Communication (SIGDOC '21), October 12-14, 2021, Virtual Event, USA. ACM, New York, NY, USA, 11 pages. https://doi.org/10.1145/3472714.3473644

\section{INTRODUCTION}

With the advancement of digital media and technology, social media use has increased ever since. Amid this trend, Facebook and WeChat have two significant populations that represent a wide variety of local and cultural contexts. While these platforms help network users, they have functioned multifaceted roles depending on users' purposes and uses. Users not only use these sites but also associate

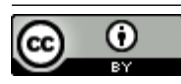

This work is licensed under a Creative Commons Attribution International 4.0 License.

SIGDOC '21, October 12-14, 2021, Virtual Event, USA

(C) 2021 Copyright held by the owner/author(s).

ACM ISBN 978-1-4503-8628-9/21/10.

https://doi.org/10.1145/3472714.3473644

\author{
Jianfen Chen \\ English Department, Purdue University, West Lafayette, \\ Indiana, USA \\ chen3421@purdue.edu
}

their identities with them. When users see their identities associated with any technological infrastructure, users and the digital technologies maintain a symbiotic relationship as users influence the design aspect of that digital technology and vice versa. Along this vein, Menendez-Blanco et al. argued that as artefacts, digital platforms help support the formation of publics and "facilitate the formation of socio-material assemblies around matters of concern" [24]. Therefore, studying any digital platform can help understand the local cultural practices, behaviors, identity, preferences, and social dynamics of that particular community. Similarly, because of the diverse cultural context, social practices, preferences, behaviors, identities, local dynamics, and purpose, not all digital platforms are the same. These platforms are guided by dissimilar design principles and target different groups of populations.

As the dominant social media platforms in their own realms, Facebook and WeChat originate from western and eastern cultural contexts respectively with distinctive development trajectories based on their respective understanding of culturally localized users' needs and experiences. Abundant research [12, 26, 33] has focused on the differences between these two leading social media platforms by examining the practices and experiences of their users, including the different antecedents affecting information sharing by users on Facebook and WeChat [26] and users' motivations and personal characteristics for using certain social media platform [14]. In spite of a great deal of research exploring the interface design features of Facebook [9, 19, 20] and WeChat [1, 3-5, 17, 21], there is a dearth of research comparing the design features, especially the interface features between these two social media platforms [27]. Studying the interface design features of these two distinctive digital platforms can inform technical communicators of the cultural considerations in interface design and encourage factoring contextualized use of technology and the sociological approach to interface design. Using Sun's Culturally Localized User Experience (CLUE) framework, this study compares the interface features of Facebook and WeChat primarily in mobile settings and adds new knowledge to understand the cultural and contextual elements in their interface design [28]. With these premises, this study delves particularly into the following areas as the first phase of our research concerning cultural usability study of Facebook and WeChat [29].

- Difference between Facebook and WeChat in terms of their respective unique design elements/functionalities.

- Culturally specific design factors revealed in Facebook and WeChat's features and functionalities.

- Design considerations revealed through the agency, technology, identity, values, ideology, structure, power, dominance, and cultural logic. 


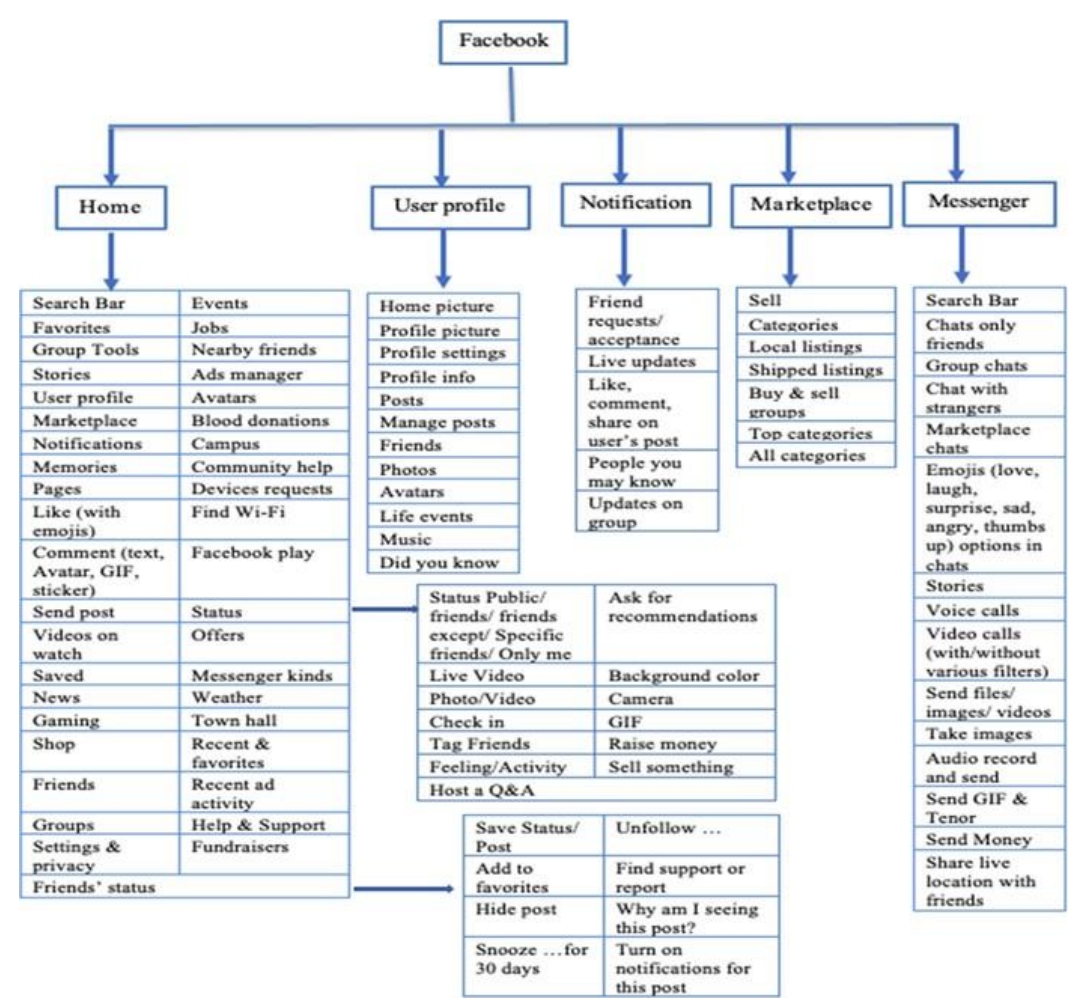

Figure 1: Interface Sketch of Facebook for Mobile Phone System

We first examine the related literature on culturally localized technology design of Facebook and WeChat. Then, we introduce CLUE [28] and Carnegie's [2] interface as exordium as the methodology, and exploratory approach as our method. Finally, we present our major findings and discuss its implication to technical communication.

\section{LITERATURE REVIEW}

In this section, we review studies related to interface design and uses of Facebook and WeChat.

\subsection{Facebook}

Facebook is the US-based SNS founded by Mark Zuckerberg in February 2004. With its wide-ranging design features (Figure 1), Facebook has been recognized as one of the biggest SNSs with more than 1.69 billion users scattered globally [30]. According to Gomes et al., "social networks have the potential to mitigate social isolation problems[...]" [9]. But not all age group people find the design aspects of any digital platform equally convenient. Although social media adoption has increased in recent years, many still are excluded because they cannot adopt them. Through a series of direct observations, interviews, and focused groups, Gomes et al.'s study identified recommendations for the design of social networks targeting seniors [9]. Based on those recommendations, they developed a prototype for tablet devices, supporting sharing and viewing Facebook content. Their user study, which compared their prototype with Facebook's native mobile application, found that
Facebook's native application does not meet senior users' concerns, like privacy and family focus, while this study's prototype, which was designed following the collected recommendations, supported relevant use cases in a usable and accessible manner.

Despite having design features that exclude a certain group of users [9], Facebook is still recognized as an SNS with a more diverse group of users compared with other SNSs originating from nonwestern contexts. For example, Li's study compared two famous SNSs, Facebook and Renren with the latter stemming from the Chinese context [19]. The study revealed multiple intriguing findings. First, Facebook users diversify the sources, while Renren network users have a limited source. Second, Facebook users have more wide groups than Renren network users, who are mainly young people aged between 18 and 34 years old. Third, the gender ratio of Facebook users is similar to men-to-women whereas Renren users are mostly women. In a similar study, Li \& Chen conducted a comparative study of Facebook and Renren's use and social capital among Chinese international students studying in the U.S [20]. This study was guided by two major questions: how the intensity of Facebook and Renren is associated with bonding social capital respectively, and whether the intensity of Facebook and Renren use have different relationships with bridging and bonding social capital respectively. The findings of the study revealed that Facebook and Renren use were linked positively bridging and bonding social capital. Meanwhile, Facebook users had a stronger relationship with bridging social capital than Renren. On the other hand, Renren users had a higher significant and positive relationship with 
maintaining home country social capital. This study resonates the significant roles local and cultural contexts play in using any SNSs. Admittedly, certain interface designs tend to function better than others as they are localized more accurately. In this juncture, our study fills this gap by studying the two SNSs that have intricately distinct origin.

In one of its rare kinds of study, Riaz and Sherani conducted a large-scale study to examine various antecedents affecting information sharing via SNSs such as Facebook and WeChat [26]. The participants of this study were international students studying at two Chinese universities, who are frequent users of these SNSs Analyzing the various variables, the findings of this study revealed no difference in the effects of perceived usefulness and information sharing attitude between Facebook and WeChat users.

Nevertheless, one finding that was significant on Facebook users only was that the positive and stronger relationship between perceived ease of use and information sharing attitude. On the other hand, the positive relationship between technological innovation and information sharing attitude was significant and stronger in WeChat users only. However, none of the studies delve into discussing the design elements or features of the two SNSs, Facebook and WeChat and their users' local cultural correlations. Being two giant SNSs, these platforms significantly engage two large populations from the western and eastern cultural and local contexts respectively. Our study focuses specifically on these SNSs' design elements, their functionalities, and their local cultural implications particularly using Sun's CLUE framework [28].

\subsection{WeChat}

Launched in 2011, WeChat is an SNS and product of China's Tencent Holdings and considered even more successful than its sibling product, QQ. WeChat is believed as the symbol of China's homegrown application and a sign that "China is moving rapidly away from relying on foreign communications technologies toward developing its own innovative formats" [11]. According to Statista [31] WeChat already has more than 1.2 billion active users across the globe. Besides mainland China, WeChat is widely used in Southeast Asian countries and countries and regions where Chinese enterprises and Chinese ethnic communities have a notable presence [18].

WeChat's unique interface contributes to the dominant use and popularity in China and beyond. Che and Ip pointed out that Xiaolong Zhang, the creator of WeChat, has always adopted minimalist design aspects for WeChat [3]. The initial interface of WeChat was characterized by only four major sections: Chats, Contacts, Discover, and Me with a search bar icon and shortcut section with a "+” sign (Figure 2). In the meantime, all expansive functions are embedded under the four main sections. The opening interface of WeChat (Figure 2) does not show the cascading and expansive functions embedded under every immediate function due to its focus on minimalist approach. Thanks to its expansive and powerful features and functions, WeChat is likened to a Swiss army knife [7] and is the embodiment and the result of Huateng Ma's envision of "convergence culture" [25]. As the founder and CEO of Tencent, $\mathrm{Ma}$ is of the view that WeChat is the SNS after QQ to achieve the convergence culture comprising six main trends of "entertainment, social networking, individual-information processing, security, internet wireless convergence, and localized application convergence" that influence Chinese Internet and Tencent strategy [25].

With WeChat penetrating people's social lives, an increasing number of studies have investigated how people use WeChat in various aspects of their daily lives. Ju and Sandel's [16] case study explored the posts on the WeChat moments of a foreign teacher living in China and analyzed the intercultural identity reflected in the posts. Their study shows that WeChat is a social media tool for internationals to acculturate the Chinese society and context. Observing people's attachment to WeChat, Cao et al. examined both technical factors of WeChat as well as intrinsic and extrinsic motivations that contribute to the additive use by WeChat users [1]. Moreover, plenty of research has focused on how users take advantage of the abundant application functions and features of WeChat in designing mobile library [8], mobile blended learning model [10], Chinese teaching programs [13, 15], and more. The above-mentioned research provides us a deeper understanding of the functions and features embedded in WeChat but does not account for the cultural considerations and factors that are in play. Bearing this in mind, in this study, we put more weight on deepening our understanding of the cultural and rhetorical factors involved in the WeChat interface that distinguish it from Facebook.

Myriad studies have highlighted the interface of WeChat from the users' perspective with each of them focusing usually on one specific feature of WeChat interface. Chen and Cheung investigated the privacy perception and protection of WeChat [4]. Their research reveals that WeChat affords the blurring boundaries between the public, professional, and private that makes WeChat unique when compared with other SNSs around the world. Chu and Zhang expounded how the group feature of WeChat offers the flexibility of establishing the group for ad hoc or long-term purposes in helping people tap and utilize three categories of social capital-bonding, bridging, and linking during the Hurricane Harvey disaster in Houston [5]. Similarly, Kow et al. compared Alipay and WeChat Wallet in their study and found these payment apps with high flexibility and extensive functions have allowed users to adaptively use digital monies [17]. In the same vein, Li et al. stated that users' intention to follow WeChat official accounts at Macao attributes to the properly designed interface and the ease of using them [21]. Our research builds on and expands the extant work on the WeChat interface by comparing it with Facebook and analyzing critically the striking features from cultural and rhetorical perspectives.

\section{METHODOLOGY AND METHOD}

Despite the wealth of research centered on user experience (UX) and interface features of SNSs, questions remain unanswered with regard to the interface features of two giant SNSs, Facebook and WeChat, given their relatively unique local and cultural contexts. Our study aims at conducting a critical comparative analysis of the interface differences between Facebook and WeChat by examining and discussing the contextual and local cultural factors that contribute to these differences. Specifically, the study intends to answer the following three primary research questions:

- What makes Facebook and WeChat different in terms of their respective unique design elements/functionalities? 


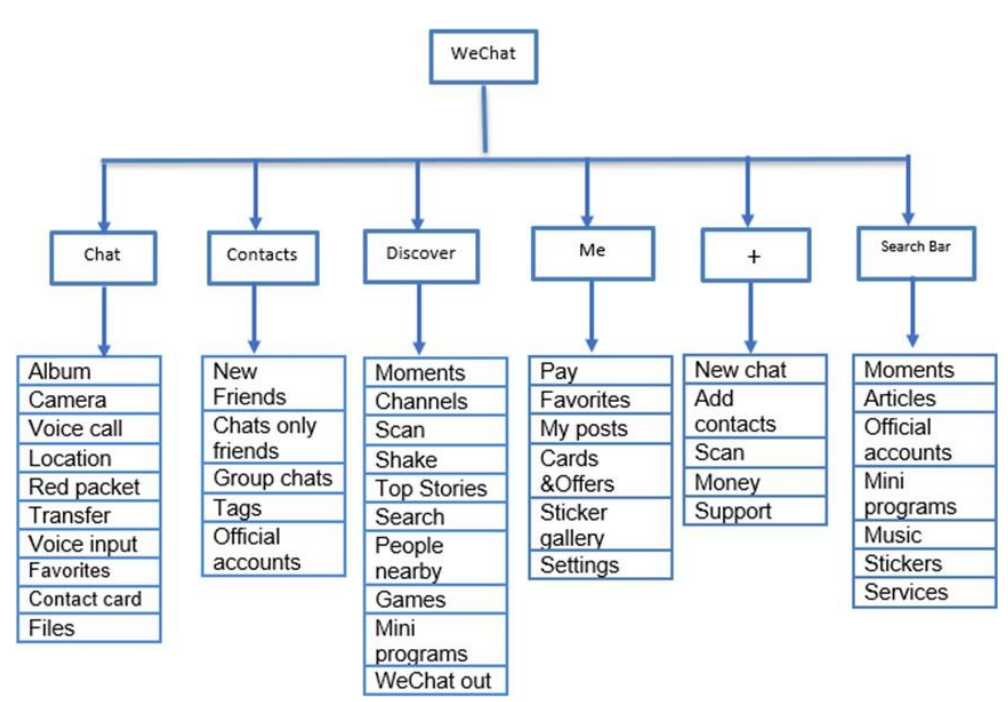

Figure 2: Interface Sketch of WeChat 7.0.21 in English Version

- What are the culturally specific design factors revealed in these features and functionalities?

- How do these features reveal the design considerations such as agency, technology, identity, values, ideology, structure, power, dominance, and cultural logic [27]?

\subsection{Methodology}

To conduct a critical comparative analysis of the interface design of Facebook and WeChat, we employed Sun's "Culturally Localized User Experience (CLUE)" framework as the first part of our methodology as we situate design feature analysis of these two sites in different cultural backgrounds [28]. Sun's work highlights the praxis of use, or "user localization that makes a usable technology meaningful to an individual" [28]. To be an accurate design, it should be contextually grounded to that particular local culture. In this sense, engaging with any particular technology reflects an element of 'identity and 'representation' of that particular culture [28]. In the meantime, local culture is not an isolated entity; instead, it is intricately connected to global culture as well. These local-global intricacies make the local design an inherent part of the globalization process [28]. Further, when she discusses the rationale to incorporate the British cultural studies in the methodology, she asserts that a technological artifact with certain design features is an assemblage of articulations between user goals and tasks, between technical functions and cultural meanings, between work efficiency and lifestyle choice, between design and production, between designer's culture and user's culture, and so on [28]. According to Sun, design is about articulation and even re-articulation of not only technical functions but also cultural meanings, a balance of work efficiency and lifestyle, and the full consideration of users' cultural and local context [28]. The three-level of affordances: operational, instrumental, and social she proposes offers a useful and practical framework for user experience analysis in cross-cultural settings [28]. In this way, designing any features of a digital device is connected with various aspects as Facebook's design features are influenced by the western cultural aspects and WeChat by the eastern cultural meanings respectively.

In addition, Carnegie's perception of the interface as exordium ("introduction" in Cicero's term) serves the second part of our methodology, since examining the rhetorical role that interface plays in new media allows us to see and talk more critically about the interface [2]. Specifically, according to Carnegie the three modes of interactivity through which interface achieves its functions are multi-directionality, level of manipulation, and presence [2]. The purpose for doing a critical rhetorical analysis of interface is to see "how the interface as exordium defines users' actions, determines the extent to which users can participate in dialogue and the creation of content and meaning, and positions users within certain social, political, economic, and cultural schemas" [2]. Basing on this analytical perspective, this study critically analyzes the interface designs of Facebook and WeChat, how users define their positions and identities when they use certain interface designs, and the socio-cultural meanings embedded with the use of these SNSs.

\subsection{Method}

To analyze the interface designs of Facebook and WeChat, we adopted an exploratory approach. Based on our personal experiences using these two SNSs and the previous studies we consulted, we arrived at focusing on four particular design aspects of Facebook and WeChat, namely, moments/status, official accounts, digital money, and group chat. Pandey has been using Facebook for over ten years, whereas Chen has been a heavy user of WeChat for ten years and a light user of Facebook for over ten years. Though both authors are not experts in each platform, our decade-long experience of using the two platforms represents experienced users and could therefore stand in for many users' experiences with Facebook and WeChat. Through frequent discussions of all interface features and functionalities between Facebook and WeChat, the two authors identified these four design features as particularly meaningful to convey the users' social and cultural practices and their identities 
embedded locally. The authors hold that these four interface functionalities bear more stark differences than other interface elements in terms of interface design than others based on their respective cultural and local contexts. As part of a larger research project, we, in this paper, present a thorough analysis and examination of these four interface design aspects as a phase one of this study. This examination also serves the basis for us to go on the second phase of this study which is working with Facebook and WeChat users to explore their cultural considerations when interacting with these interface elements.

\section{FINDINGS}

In this section, we present the key findings of our study. As highlighted in the methodology section, based on our decade-long personal experiences of using these two SNSs and previous studies, we have focused specifically on four main findings which are moments/status, official accounts, digital money, and group chat.

\subsection{Moments/Status}

Moments/status section is an important feature in many SNSs. WeChat (moment) and Facebook (status) both have this section where users update their life stories and events with their friends. Not only textual narrative, but users can also update this section with images, videos, or sharing others' posts. Users' friends can 'like', 'comment', and 'share' their posts. Besides liking any status with thumbs up, Facebook provides six emoji options to express love, care, laughter, surprise, sad, and angry attitude. WeChat on the other hand provides only two options of 'like' and 'comment' for its users to react to their friends' posts. Talking about WeChat, Che and Ip stated, "Moments is expressed in Chinese as 'Peng You Quan' (朋友圈), translated as 'friends circle”' [3]. They further mention that WeChat uses a minimalist design principle that is reflected in three different facets which are image publishing, privacy setting, and spam control [3]. Facebook has designed a timeline section to update with a prompt, "What's in your mind, [your name]?" Additionally, Facebook allows users to update with live video, feeling/activity, and stories. In this way, Facebook seems neutral giving users' equal opportunity to update their moments in any form. Users can choose their desired mode to update their timelines. On the other hand, as WeChat was initially inspired by Instagram, it encourages users to update with images rather than texts [3]. The layout of the Moments page design in WeChat is dedicated for users to use cover photos with small thumbnail images. But Facebook users' cover photo can be viewed within the user's profile page (Figure 1). "WeChat consists of a notably more image-heavy and -focused style of presentation, whereas Facebook is more expansive in its provision of a multitude of formats for submitting status and content updates" [3]. In this sense, Facebook does not prefer whether its users choose any particular type of content to update on its page, whereas WeChat seems to prefer more to the images.

WeChat Moments have some unique features that set it apart from Facebook. First, unlike Facebook, WeChat Moments is only available on mobile app. That said, if users log onto the WeChat webpage or desktop versions, they are not able to use the Moments function. Part of the reason is that WeChat limits its Moments function on the webpage and desktop version to differentiate it from Weibo which allows users to upload images or videos via the web page. Second, the Moments function offers users real-time editing features, meaning that in addition to editing images and photos, users can edit videos using stickers, typography, music, and clipping functions before posting them on the Moments section. However, despite the convenience and powerful functions of real editing that make users feel professional and confident about their posts, WeChat only allows users to upload less than 15 seconds video clips to their Moments. Nevertheless, WeChat is compatible with some video making and editing apps such as Wesee which makes it possible for users to post up to 30 seconds long video clips to their Moments. Third, the privacy setting for users' posts on the Moments is distinctive in that it offers users options to set limitations for their contacts to view their posts. With the privacy setting, the availability of the posts to users' friends varies from 'three days only' to 'always.' Like Facebook's setting for following or unfollowing posts of certain friends, WeChat Moments allows users to hide their posts from certain friends/contacts or hide the posts from certain friends/contacts. In addition, unlike Facebook, friends/contacts who are not mutual friends to a user of WeChat are not able to see, read, or respond to each other' comments on the posts of this user.

\subsection{Official Accounts}

Facebook and WeChat both have varied types of accounts for its users depending on their identities and the purpose of using these accounts. Facebook, for instance, has a general account, official page for local business or place, company, organization or institution, brand or product, artist, band or public figure, entertainment, and cause or community. Certain features of these pages remain active or play a prominent role depending on the type of account. Similar to the page function of Facebook, WeChat offers various account options too. Under the overarching name of Official Accounts, there are four types of distinctly different accounts for various groups of WeChat users (Figure 3).

Subscription Account focuses on information and brand communication. Despite its similarity to the news feed on Facebook, Subscription Account is open to both individuals such as bloggers, celebrities, and media. However, they are not as visible as news feeds on Facebook as they are nested in the subscription folder in a user's contacts.

Service Account, on the other hand, targets at businesses with a large audience and a large customer database. Functioning like a mini-business website, Service Account is characterized by some defining features, including customer service, application programming interface integration, e-commerce, and client relationship management, and so on. Unlike Subscription Account, verified service accounts can register for WeChat Pay and set up a WeChat Store.

WeChat Work (formerly known as "Enterprise Accounts") is primarily for internal communication and management. This function can be compared to Slack and Workplace by Facebook. WeChat Work has "high security as users have to follow the account and then be added manually by the account owner entering their name, phone number, and WeChat ID" [6]. Chen joined her Chinese institution's WeChat Work upon invitation and verification with her 


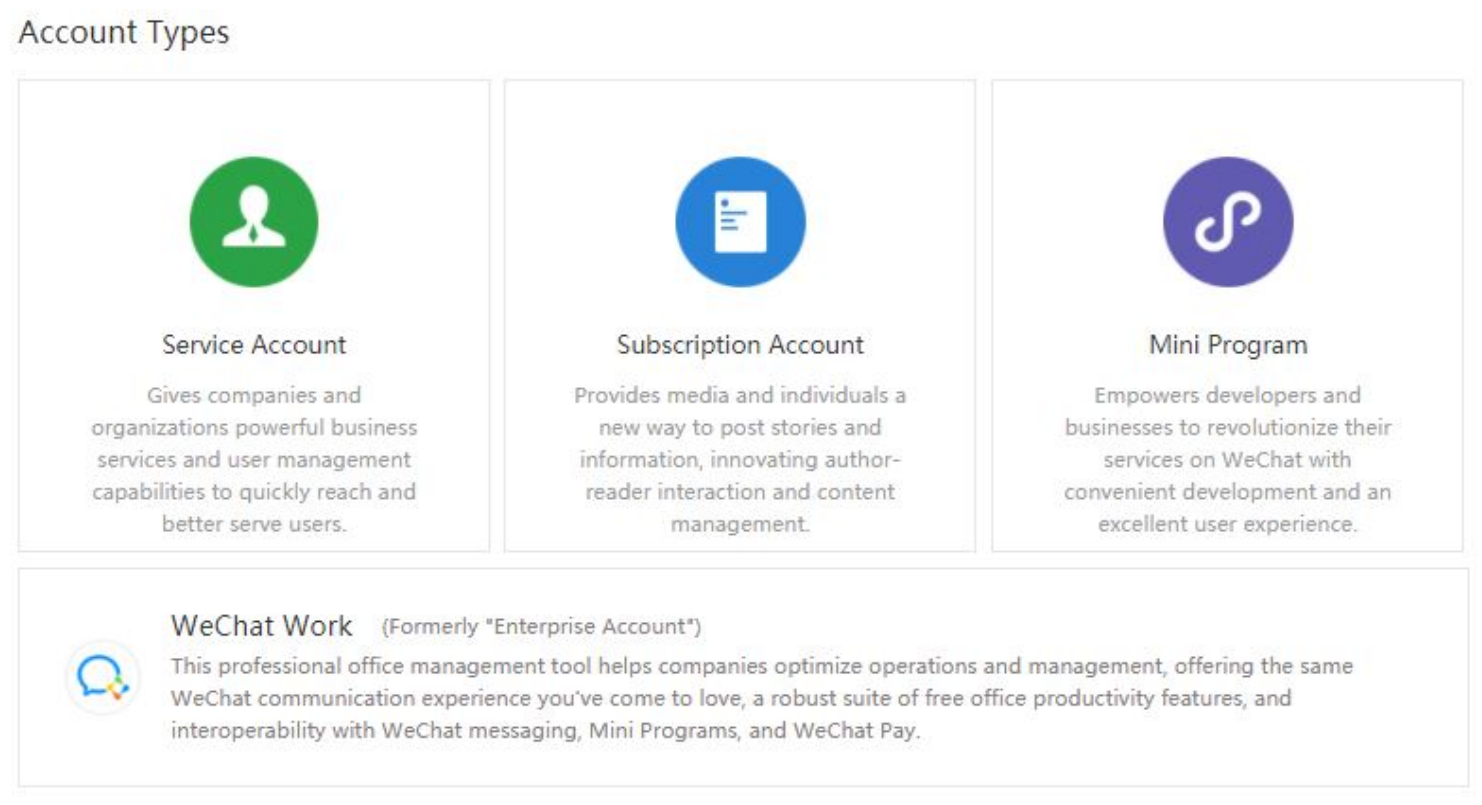

Figure 3: WeChat Official Account Types (Source: https://mp.weixin.qq.com/?lang=en_US\&token=)

university ID during the public health crisis of COVID-19. Her institution uses WeChat Work to have all staff and faculty report their health status and the virus contact history as part of the track-down measures to curb the spread of COVID-19.

The fourth type of WeChat official accounts is Mini-Program, which is "sub-applications within WeChat that allow users to access any Web app without leaving WeChat" [22]. Nested under Discovery, WeChat Mini Program does not require any installation but houses diverse programs in terms of appearances, features, and purposes. Its categories include shopping, news, games, entertainment, life, tools, education, e-commerce, travel, restaurants, library, workout, health, wealth management, social media, travel, e-government, images, job market, music, and more. Liu found that there are 2.3 million WeChat mini programs as compared with 2.1 million apps on Apple Store and 2.6 million apps on Google Play [22]. WeChat Mini Program alone competes with Google Play and Apple Store and offers programs enjoying easy access without having any hassle to download and install the standby apps. Such key features have strengthened the role of WeChat as the dominant SNS in China. For example, to control the spread of COVID-19, WeChat and Alipay have collaborated with the Chinese authorities and released a mini-program of health code to trace people's exposure risks and their mobility [23].

\subsection{Digital Currency}

One of the striking features of WeChat is making transactions with digital currency. Users can transfer money to their WeChat contacts using the 'transfer' (Figure 2) function in the chat box as long as they have money in their WeChat Pay. By connecting their bank account with their WeChat account, users can transfer money to their WeChat wallet, manage their personal funds using WeChat financial services, make payments, send money or donate to any agency via WeChat Pay using QR code. This feature has encouraged working with digital currency and connecting user's personal lives with this SNS. Because of the QR code function that WeChat has, making a payment is just a single click away from its users. In fact, digital payment is prevalent in China with WeChat Pay and other similar mobile apps like Alipay.

Unlike WeChat, Facebook's payment function is less convenient because users have to be friends to make a payment. This has limited Facebook's payment function to any specific case only (i.e., only if both the users are friends to each other) but not for any business purposes. In addition to making the payment by the users, Facebook users can also request their friends to make the payment for them (Figure 4). However, this function is limited to users in the U.S. only. Although Facebook has an option that users can connect their bank account and make any transactions, this feature has not been used extensively. Facebook does not have a $\mathrm{QR}$ code function for users to use, but they can still send money as it is one of the functions on its messenger. As a result, general users cannot make payments in any store or shopping centers. Even though users may want to use this option, it has not been as convenient as WeChat users in China. Not only this, based on our personal experiences, we have noticed Facebook users using other exclusively designed mobile apps to transfer money instead, such as Venmo, PayPal, Zelle features of any major online banking like Chase, Wells Fargo, and so on.

Figure 5 and Figure 6 display the interface of all functions available on WeChat Pay. WeChat Pay enables users to make payment for almost all their daily expenses cashless by using WeChat on their mobile phones. In addition to the conventional purpose of payment, WeChat Pay allows users to utilize the digital currencies creatively to create, build, and strengthen social cohesion and 

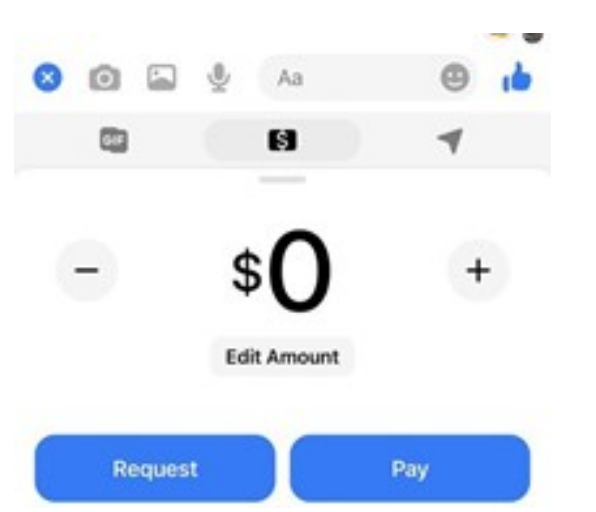

Figure 4: A screenshot of the payment function on Facebook

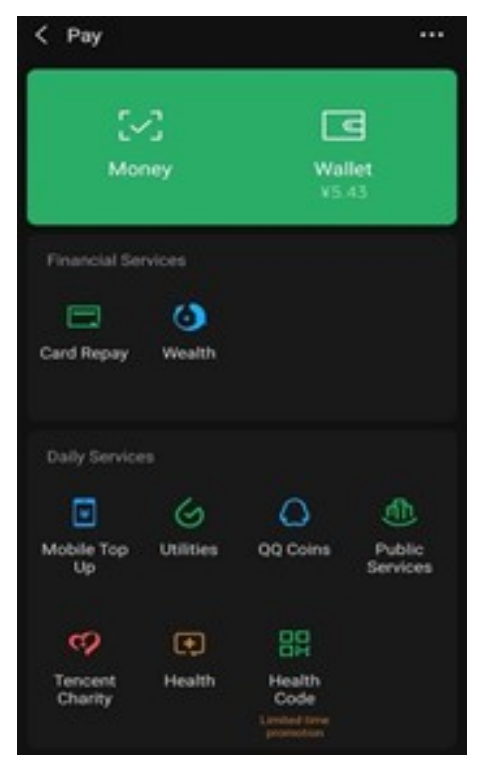

Figure 5: Screenshot of WeChat Pay (Part 1)

group values [32]. Kow et al., [17] arrived at the term, "special digital monies" to describe the special uses of digital money via WeChat in the Chinese context. This term was coined by the anthropologist Zelizer, "which suggests that attributes and meaning of a monetary transaction could change according to the specific social context" [17]. For example, WeChat Pay brings Hongbao or "red packet" (a token sum of money) as the traditional Chinese mediator of relationships between family members, close relatives, friends, and colleagues to digital spaces. For instance, senior members of the family might send Hongbao to young members as a symbol of best wishes for their life change, study progress, career promotion and so on. WeChat users also send Hongbao as a congratulatory gift to their friends on the occasions of their wedding, moving to new places, promotion, and/or other auspicious events. Interestingly, in combination with the group function in WeChat, Hongbao can be drawn on by group members and generate the "Luckiest Draw", which means a group member happens to open the red packet and gets the largest amount of token money among all draws.

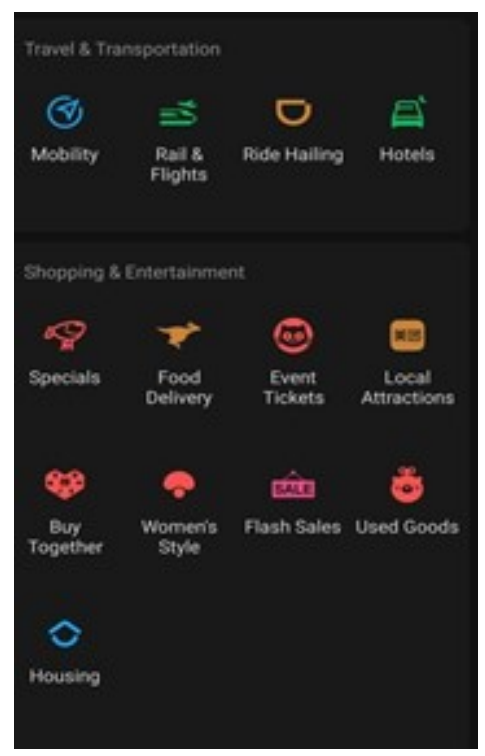

Figure 6: Screenshot of WeChat Pay (Part 2)

\subsection{Group Chat}

Group Chat is the last interface feature we analyze in this study as Facebook and WeChat have quite different functions and interfaces for Group Chat. According to Zhong, person to person and group chats are WeChat's most frequently used communication channels, whereas more public-facing walls fuel Facebook's site [34]. Dissimilar to Facebook, WeChat has no separate messenger app to facilitate group chat. People don't have to be friends with each other to join a group chat. Because of this, users of WeChat tend to be in many groups with some for ad hoc purposes and others for long-term purposes. WeChat groups can be divided roughly into groups for private businesses and groups for corporate and/or institutional businesses. Though the power dynamic and norms in different WeChat groups can vary vastly, all groups share the same interface.

Like WeChat, Facebook group chat users can name the group chat as per their own convenience. Similar to WeChat, all the users may not be mutual friends to one another, but they have to be friends of at least one member of that group to be added to that group chat. Not only on group chats, but Facebook users can also converse individually with someone who is not in their friends list but the message receiver has to approve the first message if they want to chat. Even though Facebook and WeChat share some similar group chat functions such as video, stickers, group voice calls, group video calls, gifs, file transfers, and digital money, Facebook has fewer group chat functions than WeChat. In addition, Facebook has a few functions that are unique to its messengers only, such as users can create polls in their posts. Similarly, Facebook users can undo the delivered message by unsending it or removing the message either for the sender only or to all. But unlike WeChat, the removed or undone message cannot be edited, and users have to start over to compose a new message. Users can reply or forward the specific message too. Facebook automatically saves chats. Although the 
desktop version of the messenger has a search option, the mobile system option does not have a search option, unlike WeChat.

As for group settings (Figure 7), WeChat has at least six more functions than Facebook, including "save to contacts", "search chat history", "sticky on top", "remark", "group QR code", and "group notice." By saving a group to a user's contacts, users can access the group from their contacts' page. This function helps users to share information with a number of groups. The function of "search chat history" is helpful for users in an active and large group (maximum of 500 people) to retrieve information, including group members, date, photos and videos, files, links, music, transactions, and miniprograms. "Sticky on top" allows users to give priority to groups they are most interested in by placing them on the top of their chats so that they won't miss the important information in that group. "Remark" is a function that enables users to customize the group name only visible and memorable to them. "Group QR code is available to every group and can be shared with others to invite new users into the group. "Group notice" is the function only the group administrator can use to post notifications like "to-do" to the whole group.

WeChat has four unique chat functions in its group chat interface (Figure 8), including "red packet," "group note," and "group live," and "contact card", which are unseen on Facebook group chat. But Facebook and WeChat both have "location" and "voice input." "Red packet," as discussed in the previous section, is a playful and inventive way to break the ice and promote bonding among group members. Facebook's voice input helps send audio clips. Interestingly, in addition to sending audio clips with voice input, WeChat can use voice recognition and convert audio clips into text in the group chat. Facebook and WeChat's "location" feature allows users to share their geographical place with others. WeChat's "group note" feature is an efficient way for a group admin to pin information so that group members can follow and view it anytime. Similarly, "group live" allows group members to share their live stream with group members. WeChat users can use "contact cards" to share their contacts with group members.

\section{DISCUSSION}

In this section, we discuss the findings in relation to the specific design factors revealed in interface features and functionalities of Facebook and WeChat.

\subsection{Culturally Localized Interface Design}

It can be a faulty assumption if a technical communicator thinks political factors as the only reason why Facebook and WeChat have predominantly more users in the US and China respectively. Another important reason why these SNSs are prevalent in their respective regions is that the interface designs that these sites have adopted are localized, culturally adaptive, and follow their own design philosophies. Human societies are diverse, and they have their own cultural practices that guide them in unique ways. As a result, not the same interface design or a particular function of these SNSs are equally adopted and useful in China, the USA, or elsewhere. WeChat is more adaptive to cell phone devices, and it favors images, unlike text in its moment section. This is because even as early as in 2002, "the penetration rate of mobile phones was

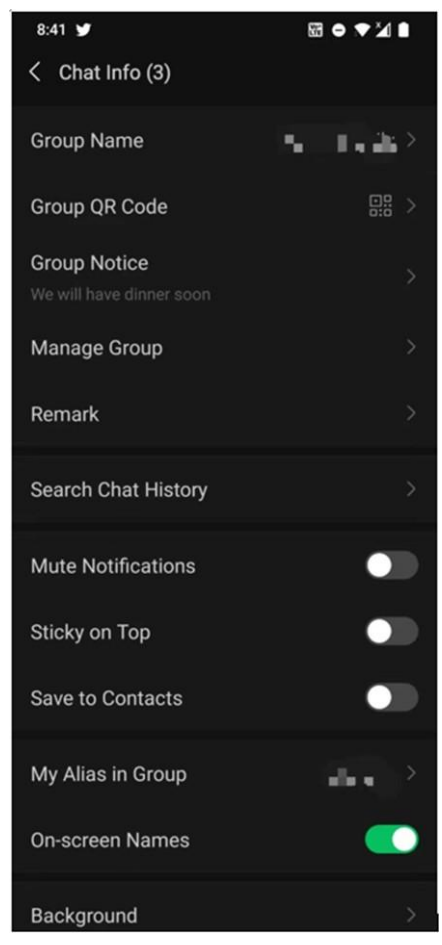

Figure 7: Screenshot of WeChat Group Setting

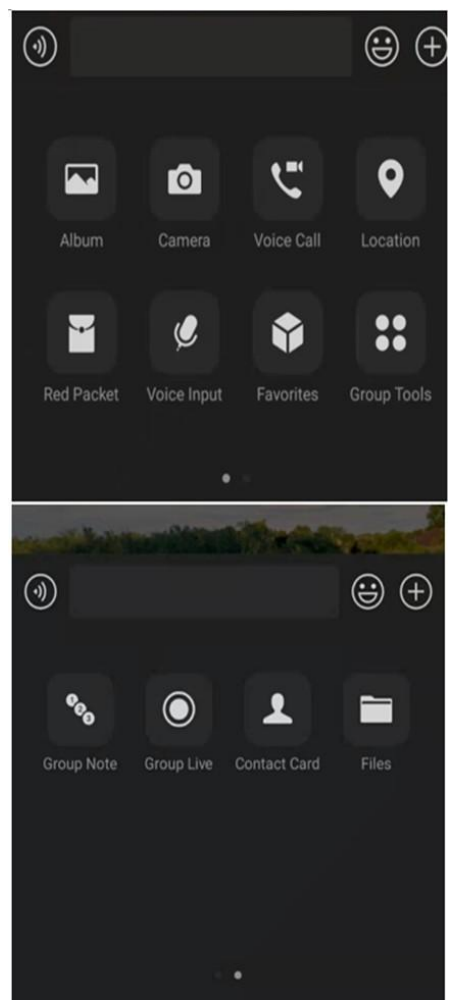

Figure 8: Screenshot of Group Chat Interface on WeChat 
eight times larger than PCs, and more than three times the number of Internet users" [28]. "In contrast, the rate of mobile phones in the U.S. was $71 \%$ of the rate of PCs, and $86 \%$ of that of Internet users" [28]. As a collective cultural practice, Chinese users tend to use WeChat for multiple purposes blurring the private/personal and professional boundaries when it comes to using WeChat features, such as making payments, sending red packets, updating personal moments, forming workmates, updating health and fitness, etc. Mostly, users seem to use Facebook for networking and socialization purposes. Next, Asians, including Chinese users, consider facesaving as an important factor in their social life. There is no agreed definition of face-saving. For this study, we understand face-saving as an action taken by users to show only their decent look, life, or events to the public to maintain the positive and esteemed profiles. Comparatively, Asian culture gives more priority to face-saving than American/western, which may explain that unlike Facebook, WeChat offers enhanced real-time editing features for users to beautify their photos and videos. Even if Facebook has some editing features while sharing stories, these features are used to a lesser extent than WeChat users do. Not sharing personal preferences publicly or in a direct manner is more of a cultural practice guided by Chinese culture. Users edit their images to hide certain elements or replace the background noise or conversation by soothing music in their video clips when they update their moments in WeChat. This is the reason why WeChat's edit functions are used more actively than Facebook. On the other hand, Facebook users do not seem to use these features much because face-saving is not a predominant culture in the U.S./west.

\subsection{Privacy Concerns and Official Accounts}

Comparatively, Facebook seems to be more liberal in terms of allowing its users to choose and create their desired account type. Depending on their purpose and identity, users can easily create their official accounts. Although Facebook advises its users to include contact information, such information is not a mandatory requirement to open an account. As the U.S. is driven by a capitalist market approach, Facebook's policy and interface design seem to be influenced by this marketing policy to some extent. Instead of strictly prohibiting users to create a certain type of account, Facebook encourages creating an appropriate page that suits users' identity and purpose so that they can best utilize certain features. However, WeChat seems to be stricter in official account opening, especially for Service Account, WeChat Work, and Mini- Program which are mostly used by enterprises or institutions. Since these official accounts are also connected with their official bank accounts to make transactions, opening accounts from outside of the mainland of China involves complicated procedures. This also entails, as Facebook does by western cultures, WeChat is influenced more by its localized Chinese cultures. Li et al. stated,

Since launching in 2012, WeChat Official Accounts has become an interactive way for content sharing and corporate marketing interaction. As of the end of 2017, there were over 20 million official accounts. WeChat Official Accounts help micro, small and medium-sized enterprises to improve operating efficiency and trim operational costs. Although the WeChat Official Account has gained a big success in China, it is not sure whether this success can be extended beyond territories. Most research about WeChat focused on the China market. [21]

Unlike the U.S., China adopts a socialist economic policy, and this might have influenced WeChat's official account opening policy in its current state to some extent. Additionally, when WeChat dominates the Chinese social media, it becomes a foreground for corporates and institutions to connect their customers or users. In a collectivist culture, people enjoy the convenience of everything in the same place. Chinese users associate their identity with such collective social and economic practices. Consequently, Chinese SNSs are guided by this culture too, which is why WeChat offers various account services targeting this entrepreneurial behavior under the name of an official account.

\subsection{SNS and Blurring Public-private Boundaries}

Using WeChat to make any payments and transactions either for a small business or any individual purposes, Chinese users' practices of SNSs embody its intricately connected personal social networking life with their business purposes. Instead of considering SNSs for networking purposes exclusively, sites like WeChat and Alipay have deeply embedded to Chinese users' lives, blurring the personal and professional boundaries. This is the reason for WeChat wallet or Alipay being famous across Chinese community [17]. The findings on digital money and digital payment practices also entail how Chinese users are transitioning to the digital age. Although digital innovations and practices are equally prevalent (if not more) in the western culture, the American culture does not accept SNSs like Facebook in the same manner that Chinese do for WeChat. One example is Facebook's less popular payment feature. Instead, multiple other digital payment options like Venmo and PayPal are in use. The reason for multiple digital payment apps being popular in the U.S. can be attributed to their desire to separate personal and professional lives. Depending on their personal preferences, users utilize other apps for payments but not as much as Chinese users do in China with WeChat or Alipay exclusively.

As detailed in the findings section, WeChat design features serve more diverse purposes than Facebook. Living in communal societies, WeChat users' multi-purposeful use of such SNS reiterates how blurred the personal and professional lives are in China. While China is shifting in its social and cultural practices with the latest digital platforms and SNSs, they accept blurred personalprofessional boundaries. The unique group chat features mirror the hierarchical layers in Chinese society and facilitate both workplace and private conversations. Conversely, Americans want to see these lines drawn distinctly, which is why Facebook's payment option has not become as much popular in the U.S. as WeChat Wallet in China. Kow et al. stated, "Alipay and WeChat Wallet are remarkable in their ability to support mobile and nuanced monetary activities, and for our informants to nearly eliminate traditional cash payments on a day-to-day basis" [17]. WeChat does not charge fees for transferring money between users, which is also part of the reason for the popularity of WeChat Pay. 


\section{IMPLICATIONS AND LIMITATIONS}

In this section, we detail the implications of the project in terms of involving culturally localized designs. Then, we reflect on the limitations of the study and share some of the possible future studies.

As the findings of this study reveal how Facebook and WeChat are influenced and inspired by its local cultures and societal needs and practices, such cultural practices are not only important for any SNS design but also for designing any technological infrastructures. Since human societies are becoming more diverse and technology is turning the human world into a global village, thinking globally and acting locally principle carries a paramount role if technical communicators want to sustain their SNSs and technological infrastructure in today's transnational business world and transcultural society. Technical communicators need to be conscious of the diverse cultural practices as our technological infrastructures represent various local cultures such as eastern vs western in case of WeChat and Facebook. How we design certain interfaces of any technology determines who our users will be, and it should also be vice versa. This is the reason why WeChat and Facebook are more popular in China and the USA than elsewhere respectively. When technical communicators have high ambition of involving as many users from as many countries or cultures as possible, they should also be considerate of how different or similar these users are in terms of their cultural practices locally. As our findings section details, face-saving culture is practiced differently in eastern and western culture. This culture also determines how they use certain interface designs of any SNSs than others. Consequently, WeChat interface designers paid more attention to the editing features in the moments section. Similarly, the prevailing gifting culture inspires the design of "red packet." Either the instance of face-saving culture or gifting via digital "red package", these practices represent the local needs and users' identities. Thus, WeChat's design features have been appropriately successful to attract more users. These instances also imply that design features fulfil their purposes when digital platforms including SNSs accurately consider when designing their features.

After analyzing the data of our study, we realized that due to its scale, this study has some inherent limitations. As the first phase of a larger research project, we relied on existing literature and our own personal user experiences to address our research questions. This might have affected the overall depths of the study in terms of data collection, literature review, data analysis, and interpretation. Thus, we reckon that having a large amount of data from more users will give very specific guidance while analyzing and interpreting the data. Because of the limited amount of data, we could only speculate the interpretations on some of our findings. If we had incorporated more data from more users, it would have more rationale coming to a particular conclusion.

In terms of future work, the next step of this study is to unpack the cultural considerations and factors underpinning the different interface features in Facebook and WeChat more specifically based on observing and surveying the actual practices of users or interviewing them. More in-depth study and more data are needed to study users' agency, technological practice, identity, values, their ideologies, social structure, power, dominance, and cultural logic in interface design. In addition to studying the two social media giants,
Facebook and WeChat, correlating our findings with other similar studies remains crucial to study transcultural and transnational users of social media designs. For this, we reckon the possibility and importance to expand this study to investigate the design features of Twitter, Instagram, QQ, and Weibo.

\section{CONCLUSIONS}

In conclusion, this study contributes to the analysis of design features of two social networking sites that represent eastern and western cultures, WeChat and Facebook. By employing a critical comparative study approach, we delved into analyzing the cultural implications of four major interface design features of these SNSs. Our study concludes that Facebook and WeChat both have been influenced by its local cultures, respectively. These findings entail that cultural and local differences are the driving force for the unique interface designs of these SNSs. Facebook's U.S.-specific payment option and WeChat's convenient official accounts service to its users of mainland China reiterate that these sites are influenced more by its local users. Thus, the design features of these sites are different, and these features are a cultural iceberg too. Studying these SNSs helps understand the local culture of that community and the vice versa. The design aspects of these features are meaningful in their own ways. Having WeChat's QR code in easily accessible places in Chinese shopping centers reveals how Chinese users have practiced their blurring personal and professional identities in their lives. On the other hand, American users connect their social media use to their professional lives less and don't make any transactions like Chinese users on WeChat, through Facebook's payment function. Moving on to the broader rhetorical implications, while any design features of these social media platforms are not permanent, studying these design features and social practices is important to identify and reflect how local cultural practices help design any technological features in a certain way.

\section{REFERENCES}

[1] Xiongfei Cao, Mingchuan Gong, Lingling Yu, and Bao Dai. 2020. Exploring the mechanism of social media addiction: an empirical study from WeChat users. Internet Research, 30(4), 1066-2243.

[2] Teena A.M. Carnegie. 2009. Interface as exordium: the rhetoric of interactivity. Computer and Composition, 26, 164-173.

[3] Xianhui Che and Barry Ip. 2017. Social networks in China. Elsevier Ltd. DOI: https://doi.org/10.1016/C2016-0-01568-1

[4] Zhen Troy Chen and Ming Cheung. 2018. Privacy perception and protection on Chinese social media: a case study of WeChat. Ethics and Information Technology, 20, 279-289. DOI:10.1111/disa.12388

[5] Tony DeGennaro. 2019. The ultimate beginner's guide to WeChat official accounts for business. Marketing in China, WeChat Marketing. https://www.dragonsocial. net/blog/beginner-guide-wechat-for- business/

[6] Thomas Graziani. 3 Oct. 2014. 2016. WeChat releases "enterprise accounts" to manage companies' internal processes. https://walkthechat.com/wechat-releasesenterprise-accounts-manage-companies-internal-processes/

[7] Dylan Dubeau. 2016. How China is changing your internet. https://www. dylandubeau.com/how-china- is-changing-your-internet-the-new-york-time

[8] Lijun Fu, Qun He, and Qingkui Xi. 2020. Evaluation of the usability of mobile library based on analytic Hierarchy process: case of WeChat library. Science \& Technology Libraries, 39(2), 155-164. DOI: 10.1080/0194262X.2020.1715322

[9] Gonçalo Gomes, Carlos Duarte, José Coelho, and Eduardo Matos. 2014. Designing a Facebook interface for senior users. The Scientific World fournal, 1-9. DOI: http://dx.doi.org/10.1155/2014/741567

[10] Yanyan Han, Liyue Tian and Weijun Cheng. 2017. Design and implementation of mobile blended learning model based on WeChat public platform. MATEC Web of Conference, 100(0202), 1-6. DOI. 10.1051/710002020

[11] Eric Harwit. 2019. WeChat and the growth of China's indigenous internet. EastWest Wire, 1-2 
[12] Hanjing Huang, Hengameh Akbaria, Nina Alef, Phairoj Liukitithara, Monica Marazzi, Bastian Verhaelen, Gina Chi-Lan Yang, and Pei-Luen Patrick Rau. 2018. International Users' Experience of Social Media: A Comparison Between Facebook and WeChat. In Rau PL. (eds), Cross-Cultural Design: Methods, Tools, and Users. CCD, pp. 341-349. Lecture Notes in Computer Science, 10911. Springer, Cham. DOI: https://doi-org.ezproxy.lib.purdue.edu/10.1007/978-3-319-921419_26

[13] Xiaoqin Huang. 2019. WeChat-based teaching for an immersion cultural exchange program - a case study in CFL. Smart Learning Environments, 6(7). 2-21. DOI: 10.1186/s40561-019-0087

[14] Linda A. Jackson and Jin-Liang Wang. 2013. Cultural differences in social networking site use: A comparative study of China and the United States. Computers in Human Behavior, 29, 910-921. DOI: http://dx.doi.org/10.1016/j.chb.2012.11.024

[15] Li Jin. (2018). Digital affordances on WeChat: learning Chinese as a second language. Computer Assisted Language Learning, 31 (1-2), 27-52. DOI:10.1080/09588221.2017.1376687

[16] Bei Ju and Todd L. Sandel. 2018. Who am I? A case study of foreigner's intercultural identity in China as presented via WeChat moments. China Media Research, 14(2), 62-74.

[17] Yong Kow, Xinning Gui, and Waikuen Cheng. 2017. Special digital monies: The design of Alipay and WeChat Wallet for mobile payment practices in China. 16th IFIP Conference on Human-Computer Interaction (INTERACT), Sep 2017, Bombay, India. pp.136-155. DOI: 10.1007/978-3-319-68059-0_9

[18] Cyruc Lee. 2019. Daily active users for WeChat exceeds 1 billion. ZDNet https://www.zdnet.com/article/daily-active-user-of-messaging-app-wechatexceeds-1-billion/

[19] Lin Li. 2011. Social network sites comparison between the united states and China: Case study on Facebook and Renren network. 2011 International Conference on Business Management and Electronic Information, 825-827. DOI: 10.1109/ICBMEI.2011.5917063

[20] Xiaoqian Li and Wenhong Chen. 2014. Facebook or Renren? A comparative study of social networking site use and social capital among Chinese international students in the United States. Computers in Human Behavior, 35, 116-123. DOI: https://doi.org/10.1016/j.chb.2014.02.012

[21] Siu Pan Li, Hoi Cheng Ip, and Jia Cheng Huang. 2019. The Use of WeChat Official Accounts in Macau: From the Customers' Perspectives. In Proceedings of the 2019 3rd International Conference on E-commerce, E-Business and E-Government (ICEEG 2019). ACM, New York, NY, USA, 49-52. DOI: https://doi.org/10.1145/ 3340017.3340023

[22] Natalie Liu. 2018. WeChat mini programs: the complete guide for business. Marketing in China. https:/www.dragonsocial.net/blog/wechat-mini-programs/
[23] Fan Liang. 2020. Covid-19 and health code: how digital platforms tackle the pandemic in China. Social Media + Society, 1-4. DOI: 10.1177/2056305120947657

[24] Maria Menendez-Blanco, Antonella De Angeli, and Maurizio Teli. 2017. Biography of a design project through the lens of a Facebook page. Computer Supported Cooperative Work, 26, 71-96. DOI: 10.1007/s10606-017-9270-4

[25] Gianluigi Negro, Gabriele Balbi, and Paolo Bory. 2020. The path to WeChat: How Tencent's culture shaped the most popular Chinese App, 1998-2011. Global Media and Communication, 16(2), 208-226. DOI: 10.1177/1742766520923008

[26] Muhammad Riaz and Sherani. 2021. Investigation of information sharing via multiple social media platforms: a comparison of Facebook and WeChat adoption. Quality and Quantity. https://doi- org.ezproxy.lib.purdue.edu/10.1007/s11135-02001079-2

[27] Huatong Sun. 2020. Global social media design: Bridging differences across cultures. New York, NY: Oxford University Press.

[28] Huatong Sun. 2012. Cross-cultural technology design: Creating culture-sensitive technology for local users. New York, NY: Oxford University Press.

[29] Huatong Sun. 2002. Exploring cultural usability. Proceedings of IEEE International Professional Communication Conference, 319-330. DOI: 10.1109/IPCC.2002.1049114

[30] Statista. (2020). Number of monthly active Facebook users worldwide as of 4th quarter 2020. https://www.statista.com/ statistics/264810/number-of-monthly-active-facebook-usersworldwide/\#: :text=How\%20many\%20users\%20does\%20Facebook,network\%20ever\%20 to $\% 20$ do $\% 20$ so.

[31] Statista. (2020). Number of monthly active WeChat users from 2nd quarter 2011 to 3rd quarter 2020. https://www. statista.com/statistics/255778/number-of-active-wechat-messengeraccounts/\#: :text=The\%20number\%20of\%20Tencent's\%20WeChat,wide\%20range $\% 20$ of $\% 20$ age $\% 20$ groups.\&text=First $\% 20$ released $\% 20 \mathrm{in} \% 202011 \% 2 \mathrm{C} \%$ 20WeChat,by\%20the\%20Chinese\%20company\%20Tencent

[32] Yang Wang and Scott D. Mainwaring. 2008. Human-Currency Interaction: learning from virtual currency use in China. In Proceedings of the SIGCHI Conference on Human Factors in Computing Systems (CHI '08). ACM, New York, NY, USA, 25-28. DOI: https://doi-org.ezproxy.lib.purdue.edu/10.1145/1357054.1357059

[33] Chunhui Xie, Jagannadha Sri Harsha Putrevu, and Chelsea Linder. 2017. Family, friends, and cultural connectedness: A comparison between WeChat and Facebook user motivation, experience and NPS among Chinese people living overseas. In: Rau PL. (eds) Cross-Cultural Design. CCD 2017. Lecture Notes in Computer Science, vol 10281. Springer, Cham.

[34] Rui Zhong. 2019. What Facebook needs to learn from WeChat's group-chat pains. Tecnode. https://technode.com/2019/03/15/what-facebook-needs-to-learn-fromwechats-group-chat-pains/ 